\title{
Natural connectivity and robustness in the topological structure of Eletrosul transmission system
}

\author{
M.S. Onetta, T. Carlotto, J.M.V. Grzybowski \\ Universidade Federal da Fronteira Sul - UFFS \\ Av. Dom João Hoffmann, 313, CEP 99.700-000, Erechim, RS. \\ E-mail: jose.grzybowski@uffs.edu.br
}

\begin{abstract}
The robustness of network structures under failure or malfunction of a subset of its nodes is recognized to be related to the number of redundant paths between nodes, which facilitate the redistribution of flows and contributes to the maintenance of its functions in the period post-failure. This paper investigates robustness on the basis of the variation of natural connectivity induced by the addition of links in a model of Eletrosul transmisson system. The results provide information regarding optimal strategies for the expansion of the electrical grid from the viewpoint of maximizing the availability of redundant closed paths within the network structure, which is believed to be related to the structural robustness of network systems.
\end{abstract}

\section{Introduction}

Electrical networks are large-scale systems whose elements feature highly interdependent behavior. This is so mainly to induce inertia which, in this context, is the inclination to remain in uniformly-paced global movement thus dissipating local instabilities. A central matter regarding electrical networks and network structures in general is the extent to which their topology can support the continuity of the processes they perform and remain operational under the failure or malfunctioning of a subset of its nodes or links. Such robustness consists of an innate structural ability to handle shortcomings, and it is recognized to be related to the availability of redundant paths connecting nodes, which facilitate the redistribution of flows after a failure. Consider previous studies which found that increased number of alternatives reduces damage in the diameter a network under attack [1]. Also, the addition of redundant links was shown to improve the tolerance against coordinated attacks [2], such that robustness is argued to increase with connectivity[3][4]. Further, diverse network systems such as water distribution networks were found to have its robustness associated to the existence of redundant paths [5].

In this regard, a measure of redundant paths in a network structure is found in the concept of natural connectivity, which is regarded as a computationally affordable and physically intuitive framework to access the number and importance of closed paths between nodes [6]. The intuition behind such relation is that the greater number of alternative paths is likely to facilitate the communication between vertices as some links or nodes are damaged [6]. As investments in power grids are subject to economical restrictions, increasing structural robustness in such networks shall obey optimality criteria. In the context of structural robustness, a question arises: how to add links such that connectivity increases the most? That is to say, how to maximize the number of redundant closed paths with a minimum addition of links?

This paper studies the impact of the addition of links upon the natural connectivity of the graph of Eletrosul transmisson system [7]. In the context of this investigation, the question being asked translates into an objective criteria, i.e., the variation of natural connectivity (VNC). The results are given in terms of VNC maps and they reveal optimal strategies for maximal connectivity increase. They also provide potentially insightful information regarding the expansion of the electrical grid towards a more and more robust and redundant topological structure.

\section{Materials and methods}

As modeled according to its topology, a power grid can be represented by a graph in which nodes (generators, substations, transformers) communicate with other nodes by means of links (transmission lines). Due to the nature of its functioning, a power grid can be modeled as a simple 
undirected graph $G(V, E)$ composed by nodes from a set $V$ which are linked by edges from a set $E$. The resulting graph induces a symmetrical adjacency matrix with entries $A_{i j}=A_{j i}=1$ if there is a link between nodes $i$ and $j$ and $A_{i j}=A_{j i}=0$, otherwise for $i, j=1, \ldots, N$, with $A_{i i}=0$. From the adjacency matrix, the network Laplacian matrix can be defined as $G=D-A$, where $D$ is a diagonal matrix for which $D_{i}=\sum_{j=1}^{N} A_{i j}$. In such a graph, a walk of length $k$ is defined as an alternating sequence of nodes and links $v_{0} e_{1} v_{1} e_{2} \ldots e_{k} v_{k}$ with $v_{i} \in V$ and $e_{i}=\left(v_{i-1}, v_{i}\right)$; further, it is closed if the walk ends in the same link as it started, that is, $v_{k}=v_{0}$. In the calculation of the natural connectivity, the weighted sum of numbers of closed walks gives shorter closed walks increased importance by dividing the number of closed walks of a given length by the factorial of the length, as given by

$$
S=\sum_{k=0}^{+\infty} \frac{n_{k}}{k !}
$$

where $n_{k}$ the number of closed walks of length $k$ can be obtained from the $k^{\text {th }}$ power of the adjacency matrix, that is,

$$
n_{k}=\sum_{i=1}^{N} \lambda_{i}^{k}=\operatorname{trace}\left(A^{k}\right)
$$

Equations (1) and (2) yield

$$
S=\sum_{k=0}^{+\infty} \frac{n_{k}}{k !}=\sum_{k=0}^{+\infty} \sum_{i=1}^{N} \frac{\lambda_{i}^{k}}{k !}=\sum_{i=1}^{N} \sum_{k=0}^{+\infty} \frac{\lambda_{i}^{k}}{k !}=\sum_{i=1}^{N} e^{\lambda_{i}}
$$

that is named Estrada index [6]. By scaling and averaging $S$, the natural connectivity of the graph is defined as

$$
\bar{\lambda}=\ln \left(\frac{1}{N} \sum_{i=1}^{N} e^{\lambda_{i}}\right)
$$

which represents an average of the eigenvalues of graph the adjacency matrix. We consider the measure in equation (4) and the adjacency matrix induced by the graph of Eletrosul transmission system [7] to evaluate the impact of the addition or removal of nodes. Towards that end, we define the VNC $\Delta \bar{\lambda}$ as

$$
\Delta \bar{\lambda}=\bar{\lambda}^{C}-\bar{\lambda}
$$

where $\bar{\lambda}^{c}$ is the natural connectivity of the graph $G(V, E \cup C)$ and we take $\bar{\lambda}$ to be the natural connectivity of the unchanged graph.

\section{Results and discussion}

Consider the original topology of the transmission system, as shown in Figure 1. Let us consider the situation in which 10 links (arbitrary) are added to the original topology in two different manners: (i) by choosing the ones that induce largest increase in VNC and then (ii) by choosing them at random. Figure 2 shows the values of the VNC corresponding to the addition of one edge between nodes $i$ and $j$ in the original graph, shown in Figure 1. Highlighted colored spots indicate links that, as created, would boost connectivity the most.

First, the links ten links that induce highest increase in VNC were added to the original network and the natural connectivity of the new network reached $\cong 2.22$, thus $\cong 0,70$ higher than the original value, which demonstrates a considerable increase in the number of alternative closed paths within the network structure, especially short ones, which are regarded as more important since they denote 'proximity' between nodes. The result is shown in Figure 3. Note that the new links encompass a small region of the network and they connect geographically close nodes, thus making them suitable for an electrical network. It can be observed that the evolution of electrical networks (as much as other infra-structure networks) conform to minimum-distance rules, i.e., new links are most likely created between nodes that are geographically close, thus making the solution feasible. 


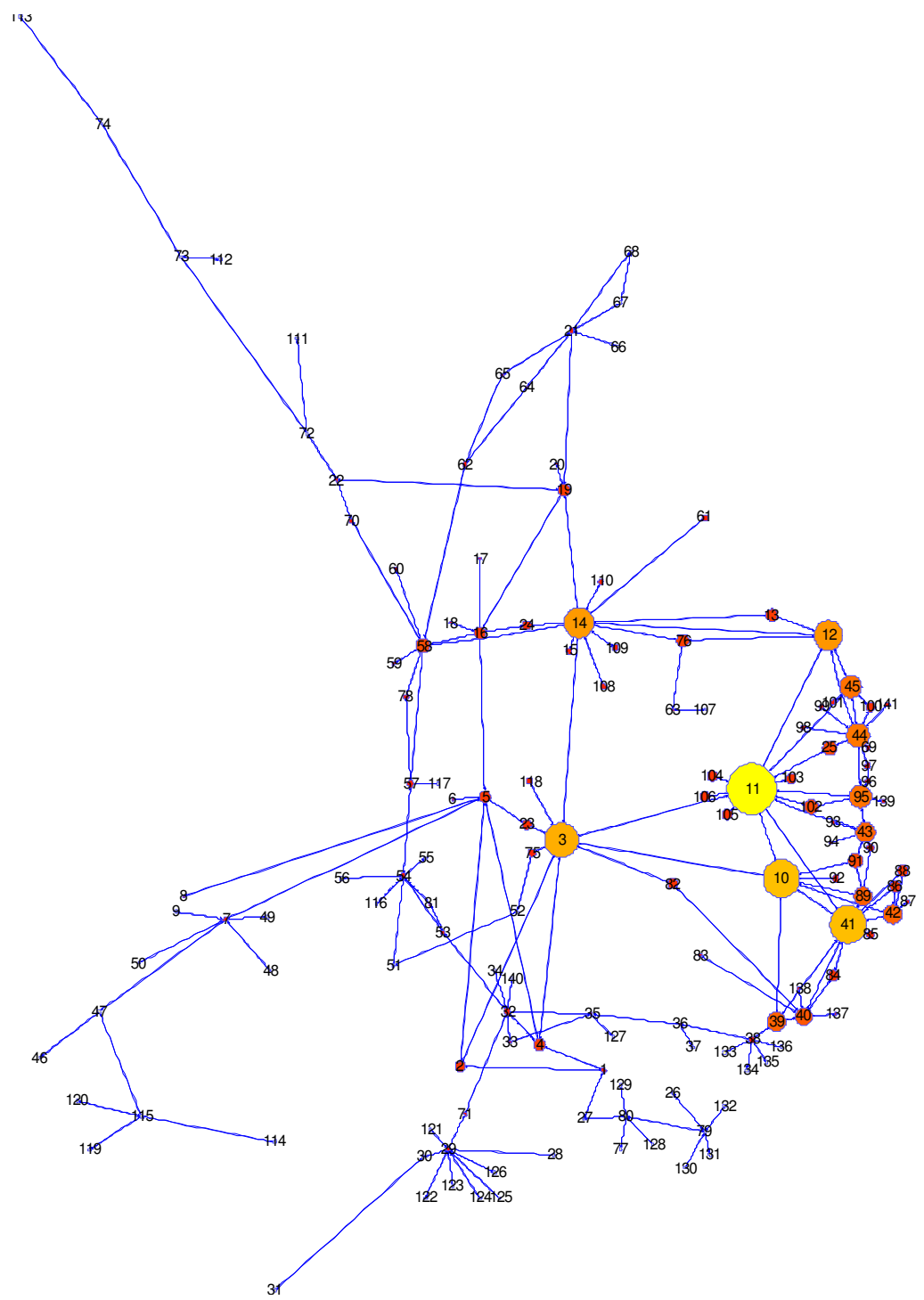

Figure 1 - Original topology of Eletrosul transmission system: nodes with major potential for connectivity increase are indicated in color gradient from red (lowest) to yellow (highest), $\bar{\lambda} \cong 1.52$.
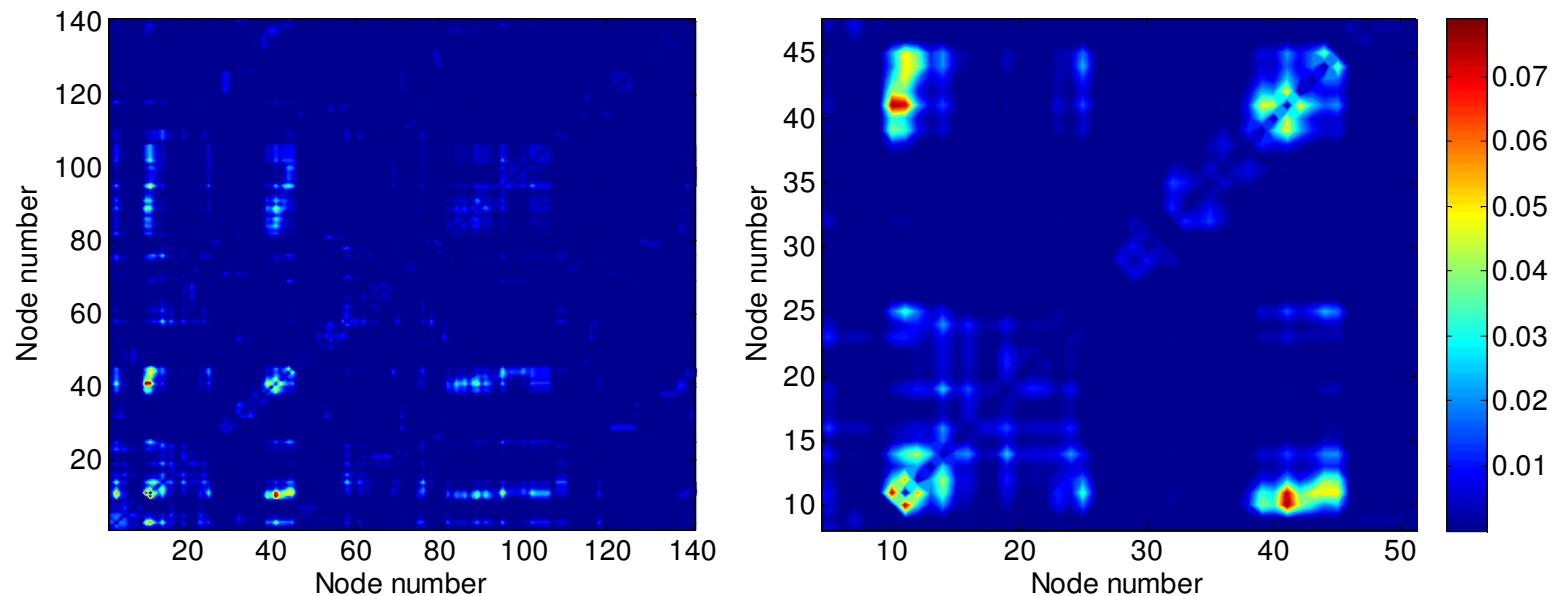

Figure 2 - Variation of natural connectivity as a function of the addition of one node: colored spots identify the position of new edges causing higher impacts upon network's natural connectivity. To the left, the contour plot for values of VNC over the set $\{1,2, \ldots, N\} \times\{1,2, \ldots, N\}$; to the right, highlight over the region with the highest values of $\mathrm{VNC}$. 


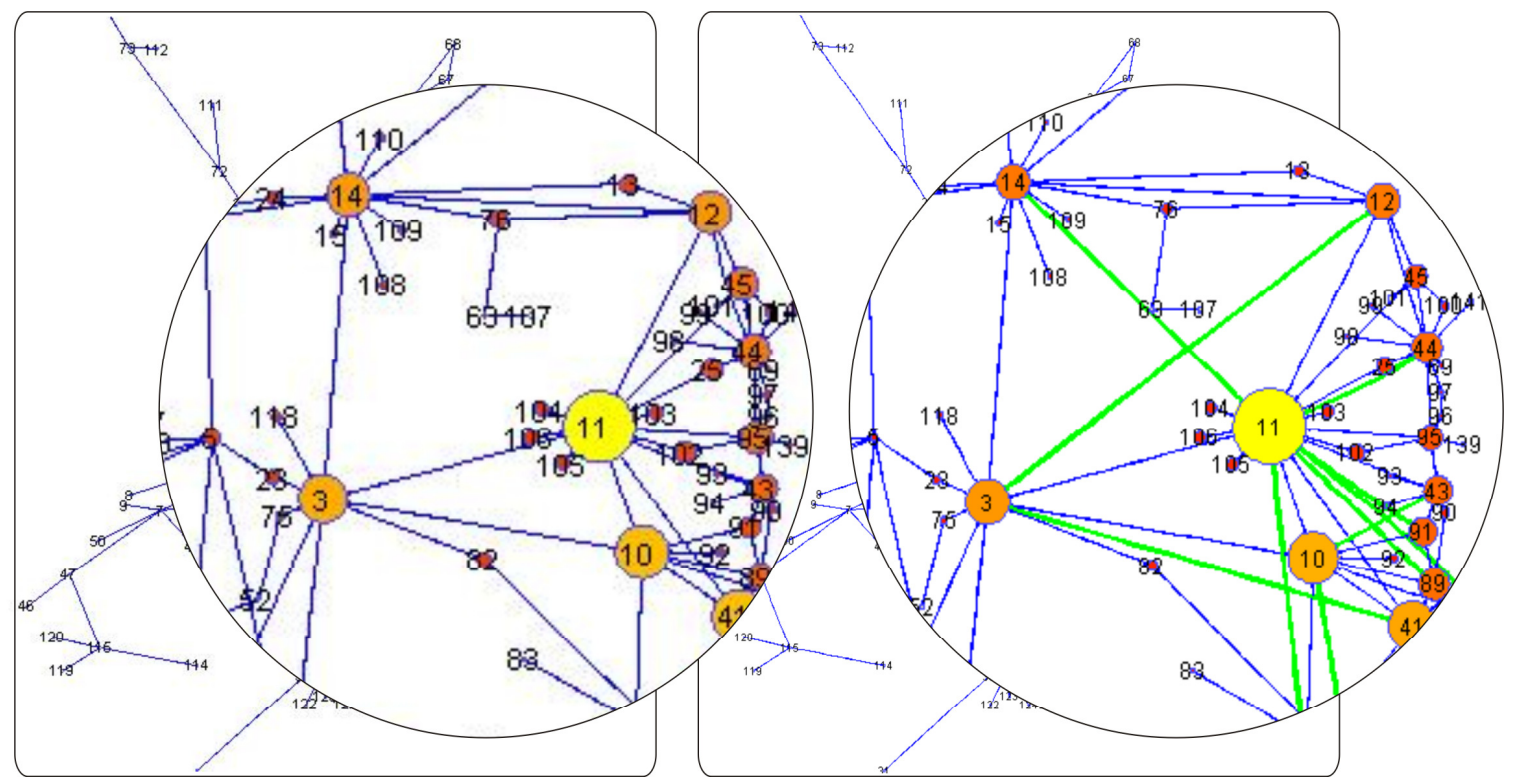

Figure 3 - Altered topology of Eletrosul transmission system after the addition of 10 new nodes that increase natural connectivity the most (in green), $\bar{\lambda} \cong 2.22$.

Second, ten random links are added to the original network and the natural connectivity of the new graph is calculated. To guarantee the uniformity in the results of the second approach, the process is repeated 1.000 times, and the results are presented as averages. The natural connectivity of the new network according to this process reached the value $\cong 1.58$, with minimum $\cong 1.55$ and maximum $\cong 1.65$ among all realizations, thus well below those obtained by directing addition by $\mathrm{VNC}$ increase.

Other than maximizing the number of alternative routes within the network structure, the proposed VNC can also be used to evaluate the impact of the removal of nodes, thus allowing identification of fragile spots within the network structure. Such information can be taken into account in priority investments regarding the improvement of the grid's structural robustness, to the extent that the results are confirmed by investigations that take into account different dimensions of the same problems, e.g., electrical, economical, technical, social, among others.

While the topology of a transmission system is an oversimplification of its general functioning, it can provide insights into the role of structure in the overly complex mechanisms that rule the functioning and failing of power grids, towards a refinement of current knowledge and understanding. From the topological viewpoint, the message conveyed by the VNC map is that increased connectivity in will improve the overall robustness of the entire network against events that may disturb the structure of the network, such as the loss of a transmission line.

The authors thank FAPERGS for the support, grant number 12/1644-2.

Keywords: natural connectivity, power-grids, robustness, Eletrosul transmission system.

\section{References}

[1] B. Shargel, H. Sayama, I. Epstein, Y. B. Optimization of Robustness and Connectivity in Complex Networks. Physical Review Letters, volume 90, number 6, 10.1103, 2003.

[2] Y. Zhuo, Y. Peng, K. Long, Y. Liu. On allocating redundancy links to improve robustness of complex communication network, Procedigs of the SPIE 7633, Network Architectures Management and Applications VII, 76331A, doi:10.1117/12.852038, 2009. 
[3] Y. Zhuo, Y. Peng, K. Long, and Y. Liu, Improving Robustness of Complex Communication Networks by Allocating Redundancy Links, in Asia Communications and Photonics Conference and Exhibition, Technical Digest (CD) (Optical Society of America), paper FD3, 2009.

[4] G. Ranjan, Z. Zhi-li, A Geometric Approach to Robustness in Complex Networks. Distributed Computing Systems Workshops, number 12137039, 2011.

[5] Yazdani, A. and Jeffrey, P. Applying Network Theory to Quantify the Redundancy and Structural Robustness of Water Distribution Systems. J. Water Resource Planning and Management, 138(2), 153-161, 2012.

[6] J. Wu, M. Barahona, Y.J. Tan, H.Z. Deng. Natural connectivity of complex networks. Chinese Physics Letters, volume 27, number 7, 078902, 2010.

[7] Map of the Electrical Transmission System, Eletrosul - Centrais Elétricas. Available online at http://www.eletrosul.gov.br/arquivos/Sistema_Transmissao_04SET2009.jpg_(2009). 